\title{
Operator Product Expansion for Form Factors
}

\author{
Amit Sever $\odot,{ }^{1}$ Alexander G. Tumanov $\odot{ }^{2}$ and Matthias Wilhelm $\oplus^{3}$ \\ ${ }^{1}$ School of Physics and Astronomy, Tel Aviv University, Ramat Aviv 69978, Israel \\ ${ }^{2}$ Max-Planck-Institut für Physik, Werner-Heisenberg-Institut, 80805 München, Germany \\ ${ }^{3}$ Niels Bohr Institute, Copenhagen University, 2100 Copenhagen $\varnothing$, Denmark
}

(Received 21 October 2020; accepted 5 January 2021; published 21 January 2021)

\begin{abstract}
We propose an operator product expansion for planar form factors of local operators in $\mathcal{N}=4 \mathrm{SYM}$ theory. This expansion is based on the dual conformal symmetry of these objects or, equivalently, the conformal symmetry of their dual description in terms of periodic Wilson loops. A form factor is decomposed into a sequence of known pentagon transitions and a new universal object that we call the "form factor transition." This transition is subject to a set of nontrivial bootstrap constraints, which are sufficient to fully determine it. We evaluate the form factor transition for maximally helicity-violating form factors of the chiral half of the stress tensor supermultiplet at leading order in perturbation theory and use it to produce operator product expansion predictions at any loop order. We match the one-loop and two-loop predictions with data available in the literature.
\end{abstract}

DOI: 10.1103/PhysRevLett.126.031602

Introduction.-The past ten years saw huge progress in our understanding of null polygonal Wilson loops, which was primarily motivated by the fact that these objects describe color-ordered scattering amplitudes in $\operatorname{planar} \mathcal{N}=$ 4 SYM theory. Another motivation lies in them controlling a certain limit of correlation functions of local operators in this theory [1-5]. A further class of fundamental observables with a dual description in terms of certain null polygonal Wilson loops are form factors (FFs) [6-10]; in terms of complexity, they lie somewhere in between scattering amplitudes and correlation functions.

The FF $\mathcal{F}_{\mathcal{O}}$ describes the overlap of a state created by a local operator $\mathcal{O}$ with an $n$-particle asymptotic state:

$\mathcal{F}_{\mathcal{O}}\left(k_{1}, \ldots, k_{n} ; q\right)=\int d x^{4} e^{-i x q}\left\langle k_{1}, \ldots, k_{n}|\mathcal{O}(x)| 0\right\rangle$,

which has support on $q=\sum_{i} k_{i}$. While $k_{i}^{2}=0$, generically $q^{2} \neq 0$. Because of the dependence on the local operator FFs are richer than scattering amplitudes, which themselves can be thought of as FFs of the identity operator. The simplest nontrivial operator to consider is the chiral half of the stress tensor supermultiplet, which contains the selfdual part of the Lagrangian, $\mathcal{F}_{\mathcal{L}}$. Operators in this multiplet preserve half of the supersymmetry and their FFs can be classified according to the helicity of the external particles.

Published by the American Physical Society under the terms of the Creative Commons Attribution 4.0 International license. Further distribution of this work must maintain attribution to the author(s) and the published article's title, journal citation, and DOI. Funded by SCOAP.
In this Letter, we focus on the simplest, maximally helicityviolating (MHV) configuration of the color-ordered form factor, $\mathcal{F}_{\mathcal{L}}^{\mathrm{MHV}}\left(k_{1}, \ldots, k_{n}\right)$, which in many ways resembles MHV scattering amplitudes.

Many of the perturbative methods for computing scattering amplitudes have been generalized to FFs, see the recent review [11] for a detailed account. Moreover, integrable structures have been identified in FFs at strong coupling $[7,12]$ as well as at weak coupling [13].

At the nonperturbative level, the only systematic method of studying scattering amplitudes is the operator product expansion (OPE), which is based on dual conformal symmetry [14]. This powerful property of planar amplitudes is nothing but the conformal symmetry of their dual description in terms of null polygonal Wilson loops. The momenta of the particles, $k_{i}$, determine the positions of the cusps of this Wilson loop by the simple rule $x_{i+1}-x_{i}=k_{i}$. For $\mathcal{F}_{\mathcal{L}}^{\mathrm{MHV}}$, the dual Wilson loop is determined in the same way. However, because the total momentum $q \neq 0$, the corresponding contour is not closed, but periodic: $x_{i+n}-x_{i}=q$. The periodicity is also imposed at the quantum level and mixes the spacetime translation with the color trace $[9,15]$. As a result, this periodic Wilson loop is only defined in the planar limit. We will also refer to it as a wrapped polygon, since it is wrapped once around a cylinder topology. Similar to amplitudes, FFs are invariant under a version of dual conformal symmetry-one that acts on both the cusps $x_{i}$ of the wrapped polygon and its periodicity constraint $[9,10,16]$. The existence of this nontrivial symmetry suggests that the OPE method can be extended to FFs. In this Letter, we present this extension explicitly. 


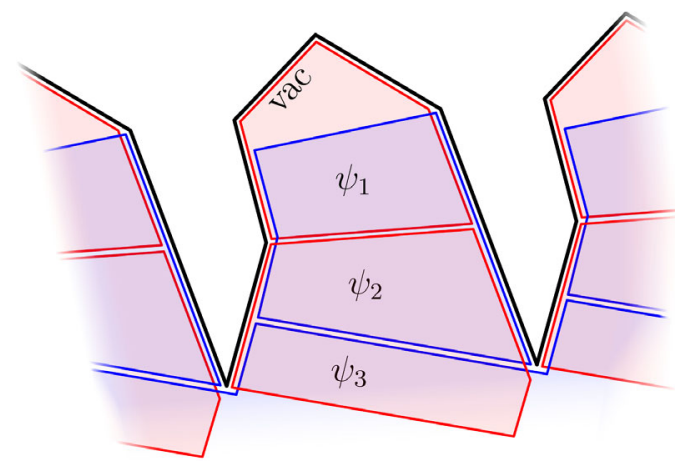

FIG. 1. Decomposition of an $n$-sided wrapped polygon into a sequence of pentagons and a two-sided wrapped polygon.

The OPE is a decomposition of the FF into two types of universal building blocks. One of them is the pentagon transition, which is independent of the operator $\mathcal{O}$ and has been bootstrapped at finite coupling in Refs. [17-26] using the integrability of the Gubser-Klebanov-Polyakov (GKP) flux tube. The other building block is the form factor transition that we introduce here, which encodes the information about $\mathcal{O}$. We also expect that it can be bootstrapped at finite coupling. One can draw an analogy between the FF OPE and the OPE for local operators in a conformal field theory (CFT). The pentagon transitions are analogous to the three-point functions, while the FF transition is analogous to the one-point function that can arise at nonzero temperature or in the presence of a defect.

Form factor OPE.-We decompose an $n$-sided wrapped polygon into one two-sided wrapped polygon and $n-2$ pentagons, which overlap on $n-2$ "middle squares," as shown in Fig. 1.

A null square is invariant under three commuting conformal symmetries; they are parametrized by $\tau, \sigma$, and $\phi$ that are conjugate to the GKP twist, conformal spin, and angular momentum in the transverse plane, respectively. We use these symmetries to parametrize all conformally inequivalent $n$-sided wrapped polygons by a set of $3 n-7$ independent conformal cross ratios as follows. To squares that are associated with an overlap of two pentagons, we assign three conformal cross ratios $\left\{\tau_{i}, \sigma_{i}, \phi_{i}\right\}_{i=1}^{n-3}$ that are defined in the same way as for closed polygons, see Fig. 2 in Ref. [17]. Similarly, for the last square the conformal cross ratios $\tau_{n-2}$ and $\sigma_{n-2}$ are defined in Fig. 2, while $\phi_{n-2}=0$.

The UV divergences of the periodic Wilson loops are regularized using the pentagons, the squares, and the twosided wrapped polygon of the decomposition. Concretely, we are considering the ratio $\mathcal{W}_{n}$ defined in Fig. 3.

The operator product expansion is the large $\tau$ expansion in which the sides of the polygon on top of each square are decomposed into a superposition of GKP flux-tube excitations. This flux is sourced by two opposite sides of the corresponding square. We start with the vacuum state in the

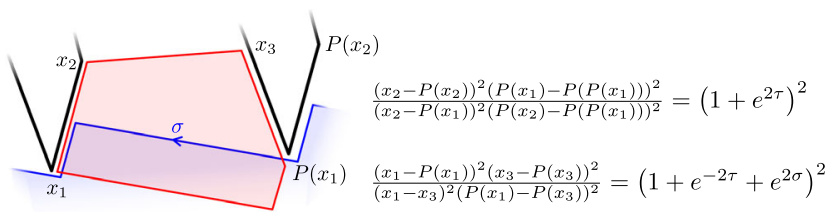

FIG. 2. We associate two independent conformal cross ratios, $\tau$ and $\sigma$, to the last OPE channel. For an $n$-particle FF, these are denoted as $\tau_{n-2}$ and $\sigma_{n-2}$. Here, $P(x)$ stands for the periodic image of the point $x$. Under a conformal transformation $x \rightarrow K(x)$, the periodic image transforms as $P(x) \rightarrow \tilde{P}(K(x))=K(P(x))$. In a conformal frame in which $P$ is a translation, $P(x)-x$ is independent of the point $x$ and the expressions for the two conformal cross ratios in the figure simplify to complete squares.

top square in Fig. 1. It undergoes a series of pentagon transitions from one square to the next, with an eigenstate in the $i$ th channel denoted as $\psi_{i}$. The propagation of this state results in the factor $\exp \left(-E_{i} \tau_{i}+i p_{i} \sigma_{i}+i m_{i} \phi_{i}\right)$, where $\left\{E_{i}, p_{i}, m_{i}\right\}$ are the GKP energy, momentum, and angular momentum, respectively. Finally, the state $\psi_{n-2}$ is absorbed by the two-sided periodic Wilson loop. We call this final step the form factor transition. In summary, this sequence of transitions and propagation can be written as

$$
\begin{aligned}
\mathcal{W}_{n}= & \sum_{\psi_{1}, \ldots, \psi_{n-2}} e^{\sum_{j}\left(-E_{j} \tau_{j}+i p_{j} \sigma_{j}+i m_{j} \phi_{j}\right)} \\
& \times \mathcal{P}\left(0 \mid \psi_{1}\right) \ldots \mathcal{P}\left(\psi_{n-3} \mid \psi_{n-2}\right) \mathcal{F}\left(\psi_{n-2}\right) .
\end{aligned}
$$

Here, $\mathcal{P}$ denotes the pentagon transition and $\mathcal{F}$ is the form factor transition.

The decomposition [Eq. (2)] applies to periodic Wilson loops in any conformal theory with a stable flux between fast-moving quarks. For the rest of this Letter we focus on $\mathcal{N}=4$ SYM theory, in which periodic Wilson loops are dual to form factors. Under this duality, the OPE maps to the expansion around the multicollinear limit. Moreover, the GKP flux-tube dynamics of this theory is integrable. Therefore, we expect to be able to bootstrap the building blocks entering Eq. (2) at finite 't Hooft coupling.

The basis of GKP eigenstates as well as their dispersion relations have been constructed in Ref. [27]. The pentagon

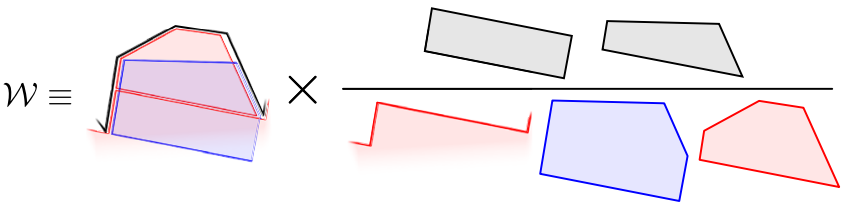

FIG. 3. A finite conformally invariant ratio is constructed by multiplying the $n$-sided wrapped polygon by all squares except for the first and dividing by all pentagons as well as the two-sided wrapped polygon, $\mathcal{W}_{n}=\left[\left\langle W_{n-\mathrm{ptFF}}\right\rangle \times\left\langle W_{2 \text { ndsquare }}\right\rangle\left\langle W_{3 \text { rdsquare }}\right\rangle \ldots /\right.$ $\left\langle W_{2-\mathrm{ptFF}}\right\rangle \times\left\langle W_{1 \text { stpentagon }}\right\rangle\left\langle W_{\text {2ndpentagon }}\right\rangle \ldots .$. . Here, this ratio is illustrated for $n=4$. 
transitions and integration (or square) measures have been bootstrapped in Refs. [17-26]. Hence, in order to compute planar form factors in $\mathcal{N}=4 \mathrm{SYM}$ theory, all that remains is to bootstrap one new building block - the form factor transition. This object is universal; it does not depend on the number of particles or their kinematical configuration, but only on the local operator and the GKP eigenstate. In the next sections, we study the FF transitions for the chiral part of the stress tensor supermultiplet. Before, let us set our notations, which are aligned with the ones introduced in Ref. [17].

The simplest form factor $\mathcal{F}_{\mathcal{L}}$ that admits a nontrivial OPE decomposition is the three-point MHV one. For this case, we have

$$
\mathcal{W}_{3}=\sum_{\mathbf{a}} \int d \mathbf{u} P_{\mathbf{a}}(0 \mid \mathbf{u}) F_{\overline{\mathbf{a}}}(\overline{\mathbf{u}}) e^{-\tau E(\mathbf{u})+i \sigma p(\mathbf{u})},
$$

where we sum over the complete basis of GKP eigenstates. These states are parametrized by the number of excitations $N$, their species $\mathbf{a}=\left\{a_{1}, \ldots, a_{N}\right\}$, and their flux-tube momenta or, equivalently, their Bethe rapidities $\mathbf{u}=\left\{u_{1}, \ldots, u_{N}\right\}$, with $\overline{\mathbf{a}}=\left\{a_{N}, \ldots, a_{1}\right\}$ and $\overline{\mathbf{u}}=\left\{-u_{N}, \ldots,-u_{1}\right\}$. Here, $P_{\mathbf{a}}$ are the pentagon transitions, and the integration measure is given by $d \mathbf{u}=$ $\mathcal{N}_{\mathbf{a}} \prod_{i=1}^{N} \mu_{a_{i}}\left(u_{i}\right)\left(d u_{i} / 2 \pi\right)$, with $\mu_{a}$ being the single-particle measures and $\mathcal{N}_{\mathrm{a}}$ being a symmetry factor. Lastly, $F_{\mathrm{a}}$ are the FF transitions that will be studied in the following two sections.

The form factor transition.-The FF transition computes the amplitude for a GKP in-state to be absorbed by the twosided wrapped polygon, see Fig. 4. It is subject to a set of constraints that we list below. These constraints are similar to those obeyed by integrable two-dimensional form factors of a branch-point operator of angle $\pi$. In Ref. [28], we use them to bootstrap the FF transitions at finite 't Hooft coupling.

Watson: Reordering two adjacent excitations within a state is equivalent to acting on it with the $S$ matrix. This property is inherited by the FF transition:

$F\left(\ldots, u_{j}, u_{j+1}, \ldots\right)=S\left(u_{j}, u_{j+1}\right) F\left(\ldots, u_{j+1}, u_{j}, \ldots\right)$,

where we have suppressed the species index.

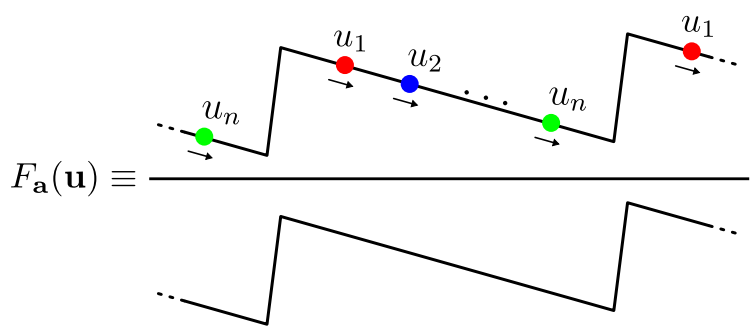

FIG. 4. The FF transition is given by the ratio between the expectation value of the two-sided wrapped polygon with and without GKP excitations inserted on its base.
Singlet: The two-sided wrapped polygon is invariant under a $U(1)_{\phi} \times S U(4)_{R}$ symmetry, where the $U(1)_{\phi}$ factor corresponds to rotations in the two-dimensional transverse plane and $S U(4)_{R}$ is the $R$-symmetry group. As a result, the FF transition must be a $U(1)_{\phi} \times S U(4)_{R}$ singlet:

$$
F_{a_{1}, \ldots, a_{n}}(\mathbf{u})=\mathcal{M}_{a_{1}}^{b_{1}} \ldots \mathcal{M}_{a_{n}}^{b_{n}} F_{b_{1}, \ldots, b_{n}}(\mathbf{u}),
$$

where $\mathcal{M} \in U(1)_{\phi} \times S U(4)_{R}$. As such, it can only absorb singlet states.

As the fundamental GKP excitations are all charged under $U(1)_{\phi} \times S U(4)_{R}$, the FF transition cannot absorb a single-particle excitation. Moreover, only singlet states with even Born-level energy can contribute to the FF transition [28]. As a result, at any loop order only even powers of $e^{-\tau}$ can appear in the large $\tau$ expansion Eq. (3).

Reflection: In addition to the continuous symmetries above, the two-sided wrapped polygon is also invariant under a discrete $\mathbb{Z}_{2}$ symmetry. It acts by flipping the direction of the two edges. This transformation has the effect of inverting the $\sigma$ direction. As a result, the FF transition is subject to the relation.

$$
F_{\mathbf{a}}(\mathbf{u})=F_{\overline{\mathbf{a}}}(\overline{\mathbf{u}}) .
$$

Square limit: The FF transition and the measure are related by

$$
\begin{aligned}
\lim _{u_{1} \rightarrow u_{n}} F_{\mathbf{a}}(\mathbf{u})= & \frac{-i \delta_{a_{n}, \bar{a}_{1}}}{\mu_{a_{1}}\left(u_{1}\right)} \frac{F_{a_{2}, \ldots, a_{n-1}}\left(u_{2}, \ldots, u_{n-1}\right)}{u_{1}-u_{n}-i \epsilon} \\
& \pm\left(S\left(u_{1}, u_{n}\right) \prod_{1<j<n} S\left(u_{1}, u_{j}\right) S\left(u_{j}, u_{n}\right)\right)_{\mathbf{a}}^{\mathbf{b}} \\
& \times \frac{-i \delta_{b_{n}, \bar{b}_{1}}}{\mu_{b_{1}}\left(u_{1}\right)} \frac{F_{b_{2}, \ldots, b_{n-1}}\left(u_{2}, \ldots, u_{n-1}\right)}{u_{n}-u_{1}-i \epsilon}
\end{aligned}
$$

where the plus sign is for bosons and the minus sign for fermions. This relation represents a factorization limit in which a pair of excitations decouples from the rest [28].

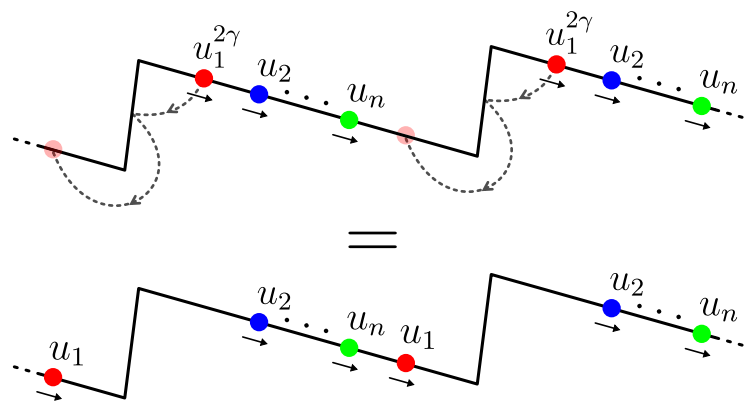

FIG. 5. Applying a mirror transformation to the first excitation is equivalent to transporting it to the neighboring edge on the left. After two successive mirror transformations, or a crossing transformation, the first excitation becomes the last one. 
Crossing: The most nontrivial constraint has to do with the crossing symmetry of the transition and is depicted in Fig. 5. It reads

$$
F\left(u_{1}^{2 \gamma}, u_{2}, \ldots, u_{n}\right)=F\left(u_{2}, \ldots, u_{n}, u_{1}\right) .
$$

Here, $u^{\gamma}$ is a mirror transformation such that $p\left(u^{\gamma}\right)=i E(u)$ and $E\left(u^{\gamma}\right)=i p(u)$, see Ref. [27].

FF transitions at Born level.-The leading contribution to the OPE Eq. (3) comes from the lightest singlet state. In perturbation theory, this contribution stems from three two-particle singlet states and two one-particle effective excitations, but the latter do not contribute at leading order [29]. All of them have the same tree-level energy $E=2$. Each of the three two-particle singlet states is a superposition of all possible singlet combinations of two scalar $(\phi \bar{\phi})$, two fermion $(\psi \bar{\psi})$, and two gluon $(F \bar{F})$ fields inserted on the base of the wrapped polygon in Fig. 4. These states differ in the asymptotic limit, in which the two fields are taken far apart. Only one out of the three pairs of fields survives in this limit, and this is the pair that labels the state.

In Ref. [29], we have explicitly constructed the aforementioned superpositions that correspond to the three twoparticle singlet states at Born level. We will now use them to compute the Born-level FF transitions we denote by $F_{\phi \bar{\phi}}$, $F_{\psi \bar{\psi}}$, and $F_{F \bar{F}}$.

At leading order in perturbation theory, the expectation value of the two-sided wrapped polygon is equal to 1 . Hence, only the numerator in Fig. 4 contributes to the transition nontrivially. Consider the wrapped polygon with two conjugate fields inserted at positions $\sigma_{1}$ and $\sigma_{2}$ with $\sigma_{2}>\sigma_{1}$. At Born level, we obtain the propagator between the field at $\sigma_{2}$ and the periodic image of the field at $\sigma_{1}$ :

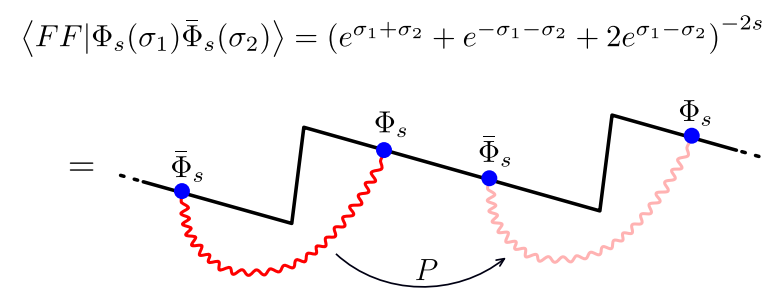

where $\Phi_{s}$ is a field of conformal spin $s=\frac{1}{2}, 1, \frac{3}{2}$ for scalars, fermions, and gluons, respectively.

Convoluting the singlet states given in Ref. [29] with the propagator Eq. (9), we arrive at

$$
\begin{aligned}
F_{\phi \bar{\phi}}(u, v)= & -\frac{4}{g^{2}(u-v-2 i)(u-v-i)} \\
& \times \frac{\Gamma(i u-i v)}{\Gamma\left(\frac{1}{2}+i u\right) \Gamma\left(\frac{1}{2}-i v\right)}, \\
F_{\psi \bar{\psi}}(u, v)= & +\frac{2}{g^{2}} u \sinh (\pi u) \delta(u-v), \\
F_{F \bar{F}}(u, v)= & -\frac{2}{g^{2}}\left(u^{2}+\frac{1}{4}\right) \cosh (\pi u) \delta(u-v),
\end{aligned}
$$

where $g^{2}=\left[\left(g_{\mathrm{YM}}^{2} N\right) /\left(16 \pi^{2}\right)\right]$. Note that for two gluons and two fermions, the right-hand side of Eq. (7) reduces to a delta function divided by the measure. We see that for these states the full Born level result is given solely by this simple square limit contribution. This might be surprising, because the contribution of each type of fields to Eq. (10) is highly nontrivial, but they combine to an almost trivial result.

Perturbative tests and predictions.-We now perform a perturbative test of the FF OPE and use it to make higher loop predictions.

We start by extracting the OPE data from previously computed form factors [8]. At one-loop order, we find the ratio $\mathcal{W}_{n=3}$ defined in Fig. 3 to be given by

$$
\begin{aligned}
\mathcal{W}_{3}^{(1)}= & 4 \sigma^{2}-2 \operatorname{Li}_{2}\left(-e^{-2 \tau}\right)+2 \operatorname{Li}_{2}\left(-e^{-2 \tau}-e^{2 \sigma}\right) \\
& +2 \operatorname{Li}_{2}\left[-e^{-2 \tau}-e^{-2 \sigma}\left(1+e^{-2 \tau}\right)^{2}\right]+\frac{\pi^{2}}{3}
\end{aligned}
$$

where $\mathcal{W}_{3}=1+\sum_{\ell=1}^{\infty} g^{2 \ell} \mathcal{W}_{3}^{(\ell)}$. As expected from the singlet axiom, the large $\tau$ expansion of $\mathcal{W}_{3}^{(1)}$ contains only even powers of $e^{-\tau}$, with the leading one given by

$$
\begin{aligned}
\mathcal{W}_{3}^{(1)}= & 2 e^{-2 \tau}\left[1-2 \sigma e^{-2 \sigma}-4 \cosh ^{2}(\sigma) \log \left(1+e^{-2 \sigma}\right)\right] \\
& +O\left(e^{-4 \tau}\right) .
\end{aligned}
$$

On the OPE side, we insert the Born-level FF transitions Eq. (10) into Eq. (3) and perform the integration over the two rapidities, finding a perfect match with Eq. (12).

Even without the higher loop FF transitions, we can already make certain all-loops predictions. Namely, at $\ell$-loop order we can predict the term with the highest power of $\tau$, i.e., $\tau^{\ell-1} e^{-2 \tau}$. It is given by pulling down $(\ell-1)$ powers of the one-loop correction to the energy $g^{2}\left[E_{2 s}^{(1)}\left(u_{1}\right)+\right.$ $\left.E_{2 s}^{(1)}\left(u_{2}\right)\right]$ from the exponent $e^{-\tau\left[E_{2 s}\left(u_{1}\right)+E_{2 s}\left(u_{2}\right)\right]}$. The one-loop correction to the energy of the individual excitations is given by $E_{2 s}^{(1)}(u)=2[\psi(s+i u)+\psi(s-i u)-2 \psi(1)]$, where $\psi(x)=\left[\Gamma^{\prime}(x) / \Gamma(x)\right]$ is Euler's digamma function [27].

With the two-loop data available for the three-point form factor reminder function $\mathcal{R}_{3}$ [30], we can test the OPE prediction for the $\tau e^{-2 \tau}$ term in $\mathcal{W}_{3}$ at two-loop order. To do so, one first has to translate between these two finite dual conformally invariant functions, $\mathcal{R}$ and $\mathcal{W}$. They are related 
as $\mathcal{W}_{n}=\exp \left[\frac{1}{4} \Gamma_{\text {cusp }} \mathcal{W}_{n}^{(1)}\right] \times \mathcal{R}_{n}$, where $\Gamma_{\text {cusp }}=4 g^{2}+\cdots$ is the cusp anomalous dimension. Using this relation, we obtain the following result:

$$
\begin{aligned}
\mathcal{W}_{3, \tau e^{-2 \tau}}^{(2)}= & -8\left[1-\left(1+e^{-2 \sigma}\right) \log \left(1+e^{2 \sigma}\right)\right] \\
& \times\left[1-\left(1+e^{2 \sigma}\right) \log \left(1+e^{-2 \sigma}\right)\right],
\end{aligned}
$$

which is in perfect agreement with the OPE prediction.

At three-loop order, we can predict the term proportional to $\tau^{2} e^{-2 \tau}$ :

$$
\begin{aligned}
& \mathcal{W}_{3, \tau^{2} e^{-2 \tau}}^{(3)} \\
& =\frac{16}{3} \cosh ^{2}(\sigma) \log \left(1+e^{-2 \sigma}\right)\left\{12 \sigma(4-3 \sigma)-24-\pi^{2}\right. \\
& \left.\quad+8 \log \left(1+e^{-2 \sigma}\right)\left[3-6 \sigma-2 \log \left(1+e^{-2 \sigma}\right)\right]\right\}+\frac{4 \pi^{2}}{3} \\
& \quad+24-16 \sigma\left(4-3 \sigma+4 e^{-2 \sigma}\right)-32 \cosh ^{2}(\sigma) \operatorname{Li}_{3}\left(-e^{-2 \sigma}\right) .
\end{aligned}
$$

Similarly, we can produce higher loop predictions; we refrain from giving these explicitly due to their size.

Discussion.-In this Letter, we have introduced an operator product expansion for form factors in planar $\mathcal{N}=$ 4 SYM theory. It reduces the computation of the dual periodic Wilson loop to known fundamental building blocks $[17,19]$ and a single new universal building block - the FF transition.

We have calculated the two-particle FF transition at Born level, Eq. (10). A natural finite-coupling ansatz for the gluonic and fermionic two-particle FF transitions that is consistent with all the constraints is

$$
F_{\Phi \bar{\Phi}}(u, v) \propto \frac{\delta\left[p_{\Phi}(u)-p_{\Phi}(v)\right]}{\mu_{\Phi}(u)} \times \frac{\partial p_{\Phi}(u)}{\partial u}
$$

where $\Phi \in\{\psi, F\}$ and $p_{\Phi}(u)$ is the GKP momentum. Based on this conjecture, the bootstrap constraints for the FF transition that we formulated in this Letter, and the perturbative data available to us, we were able to fix the remaining scalar two-particle FF transition at finite coupling [28]. Transitions involving more than two flux-tube excitations can hopefully be fixed in terms of the twoparticle ones using integrability. Our construction therefore opens the door for finite-coupling computations of FFs.

There are multiple future directions to pursue, some of which we list below. (i) At strong coupling, the FFs are computed by minimizing the area of a periodic string in $\mathrm{AdS}_{5}[7,12]$. We expect the corresponding Yang-Yang functional to be constructed from the gluon and fermion FF transitions, Eq. (15), along with the corresponding pentagon transitions and measures. (ii) In this Letter, we have only considered MHV FFs of the chiral part of the stress tensor supermultiplet. It would be interesting to extend our considerations to the $\mathrm{N}^{k} \mathrm{MHV}$ case, for which the result is expected to be given by a version of the superperiodic Wilson loop introduced in Ref. [9]. In parallel, it would be interesting to bootstrap the corresponding charged FF transitions, in analogy to the charged pentagon transitions of Refs. [21,22]. (iii) Another interesting direction is to consider local operators other than the chiral part of the stress tensor supermultiplet; corresponding FFs have been studied in Refs. [31-41]. $T$ duality is expected to map their higher integrability Yangian charges into dual ones $[42,43]$ that are evaluated along one period of the dual Wilson loop. (iv) It is possible to extend the hexagon function program of Refs. [44-47] to analogous FF functions [48]. The interplay between the OPE and these FF functions provides a plethora of valuable checks of our predictions and vice versa. (v) Finally, it would be interesting to see if our considerations can be used for studying FFs in other theories like Aharony-BergmanJafferis-Maldacena theory [49].

We are very grateful to B. Basso for many valuable discussions and comments on the draft. A. T. and M. W. are grateful to CERN for hospitality. A. S. is grateful to Niels Bohr Institute for hospitality. A. S. was supported by the I-CORE Program of the Planning and Budgeting Committee, The Israel Science Foundation (Grant No. 1937/12), and by the Israel Science Foundation (Grant No. 1197/20). A. T. received funding from the European Research Council (ERC) under the European Unions Horizon 2020 research and innovation programme, Novel structures in scattering amplitudes (Grant Agreement No. 725110). M. W. was supported in part by the ERC starting Grant No. 757978 and the research Grants No. 00015369 and No. 00025445 from Villum Fonden.Please review the funding information section of the proof's cover letter and respond as appropriate. We must receive confirmation that the funding agencies have been properly identified before the article can publish.

[1] L. F. Alday, B. Eden, G. P. Korchemsky, J. Maldacena, and E. Sokatchev, From correlation functions to Wilson loops, J. High Energy Phys. 09 (2011) 123.

[2] B. Eden, G. P. Korchemsky, and E. Sokatchev, From correlation functions to scattering amplitudes, J. High Energy Phys. 12 (2011) 002.

[3] B. Eden, G. P. Korchemsky, and E. Sokatchev, More on the duality correlators/amplitudes, Phys. Lett. B 709, 247 (2012).

[4] B. Eden, P. Heslop, G. P. Korchemsky, and E. Sokatchev, The super-correlator/super-amplitude duality: Part I, Nucl. Phys. B869, 329 (2013).

[5] B. Eden, P. Heslop, G. P. Korchemsky, and E. Sokatchev, The super-correlator/super-amplitude duality: Part II, Nucl. Phys. B869, 378 (2013). 
[6] L. F. Alday and J. Maldacena, Comments on gluon scattering amplitudes via AdS/CFT, J. High Energy Phys. 11 (2007) 068.

[7] J. Maldacena and A. Zhiboedov, Form factors at strong coupling via a Y-system, J. High Energy Phys. 11 (2010) 104.

[8] A. Brandhuber, B. Spence, G. Travaglini, and G. Yang, Form factors in $\mathcal{N}=4$ super Yang-Mills and periodic Wilson loops, J. High Energy Phys. 01 (2011) 134.

[9] R. Ben-Israel, A. G. Tumanov, and A. Sever, Scattering amplitudes-Wilson loops duality for the first non-planar correction, J. High Energy Phys. 08 (2018) 122.

[10] L. Bianchi, A. Brandhuber, R. Panerai, and G. Travaglini, Dual conformal invariance for form factors, J. High Energy Phys. 02 (2019) 134.

[11] G. Yang, On-shell methods for form factors in $\mathcal{N}=4$ SYM and their applications, Sci. China Phys. Mech. Astron. 63, 270001 (2020).

[12] Z. Gao and G. Yang, Y-system for form factors at strong coupling in $\mathrm{AdS}_{5}$ and with multi-operator insertions in $\mathrm{AdS}_{3}$, J. High Energy Phys. 06 (2013) 105.

[13] R. Frassek, D. Meidinger, D. Nandan, and M. Wilhelm, Onshell diagrams, Gramannians and integrability for form factors, J. High Energy Phys. 01 (2016) 182.

[14] J. M. Drummond, J. Henn, G. P. Korchemsky, and E. Sokatchev, Conformal Ward identities for Wilson loops and a test of the duality with gluon amplitudes, Nucl. Phys. B826, 337 (2010).

[15] A. Cavaglia, D. Grabner, N. Gromov, and A. Sever, Colourtwist operators. Part I. Spectrum and wave functions, J. High Energy Phys. 06 (2020) 092.

[16] D. Chicherin, J. M. Henn, and E. Sokatchev, Implications of nonplanar dual conformal symmetry, J. High Energy Phys. 09 (2018) 012.

[17] B. Basso, A. Sever, and P. Vieira, Spacetime and Flux Tube S-Matrices at Finite Coupling for $\mathcal{N}=4$ Supersymmetric Yang-Mills Theory, Phys. Rev. Lett. 111, 091602 (2013).

[18] B. Basso, A. Sever, and P. Vieira, Space-time S-matrix and Flux tube S-matrix II. Extracting and Matching Data, J. High Energy Phys. 01 (2014) 008.

[19] B. Basso, A. Sever, and P. Vieira, Space-time S-matrix and Flux-tube S-matrix III. The two-particle contributions, J. High Energy Phys. 08 (2014) 085.

[20] B. Basso, A. Sever, and P. Vieira, Space-time S-matrix and Flux-tube S-matrix IV. Gluons and fusion, J. High Energy Phys. 09 (2014) 149.

[21] B. Basso, J. Caetano, L. Cordova, A. Sever, and P. Vieira, OPE for all helicity amplitudes, J. High Energy Phys. 08 (2015) 018.

[22] B. Basso, J. Caetano, L. Cordova, A. Sever, and P. Vieira, OPE for all helicity amplitudes II. Form factors and data analysis, J. High Energy Phys. 12 (2015) 088.

[23] B. Basso, A. Sever, and P. Vieira, Hexagonal Wilson loops in planar $\mathcal{N}=4 \mathrm{SYM}$ theory at finite coupling, J. Phys. A 49, 41LT01 (2016).

[24] A. V. Belitsky, Nonsinglet pentagons and NMHV amplitudes, Nucl. Phys. B896, 493 (2015).

[25] A. V. Belitsky, Fermionic pentagons and NMHV hexagon, Nucl. Phys. B894, 108 (2015).

[26] A. V. Belitsky, Matrix pentagons, Nucl. Phys. B923, 588 (2017).
[27] B. Basso, Exciting the GKP string at any coupling, Nucl. Phys. B857, 254 (2012).

[28] A. Sever, A. G. Tumanov, and M. Wilhelm, An operator product expansion for form factors III (to be published).

[29] A. Sever, A. G. Tumanov, and M. Wilhelm, An operator product expansion for form factors II (to be published).

[30] A. Brandhuber, G. Travaglini, and G. Yang, Analytic twoloop form factors in $\mathcal{N}=4$ SYM, J. High Energy Phys. 05 (2012) 082.

[31] O. T. Engelund and R. Roiban, Correlation functions of local composite operators from generalized unitarity, J. High Energy Phys. 03 (2013) 172.

[32] A. Brandhuber, B. Penante, G. Travaglini, and C. Wen, The last of the simple remainders, J. High Energy Phys. 08 (2014) 100.

[33] M. Wilhelm, Amplitudes, form factors and the dilatation operator in $\mathcal{N}=4$ SYM theory, J. High Energy Phys. 02 (2015) 149.

[34] D. Nandan, C. Sieg, M. Wilhelm, and G. Yang, Cutting through form factors and cross sections of non-protected operators in $\mathcal{N}=4$ SYM, J. High Energy Phys. 06 (2015) 156.

[35] F. Loebbert, D. Nandan, C. Sieg, M. Wilhelm, and G. Yang, On-shell methods for the two-loop dilatation operator and finite remainders, J. High Energy Phys. 10 (2015) 012.

[36] A. Brandhuber, M. Kostacinska, B. Penante, G. Travaglini, and D. Young, The SU(2|3) dynamic two-loop form factors, J. High Energy Phys. 08 (2016) 134.

[37] F. Loebbert, C. Sieg, M. Wilhelm, and G. Yang, Two-loop SL(2) form factors and maximal transcendentality, J. High Energy Phys. 12 (2016) 090.

[38] S. Caron-Huot and M. Wilhelm, Renormalization group coefficients and the S-matrix, J. High Energy Phys. 12 (2016) 010.

[39] P. Banerjee, P. K. Dhani, M. Mahakhud, V. Ravindran, and S. Seth, Finite remainders of the Konishi at two loops in $\mathcal{N}=4$ SYM, J. High Energy Phys. 05 (2017) 085.

[40] T. Ahmed, P. Banerjee, P. K. Dhani, N. Rana, V. Ravindran, and S. Seth, Konishi form factor at three loops in $\mathcal{N}=4$ supersymmetric Yang-Mills theory, Phys. Rev. D 95, 085019 (2017),

[41] A. Brandhuber, M. Kostacinska, B. Penante, and G. Travaglini, $\operatorname{Tr}\left(F^{3}\right)$ supersymmetric form factors and maximal transcendentality Part I: $\mathcal{N}=4$ super Yang-Mills, J. High Energy Phys. 12 (2018) 076.

[42] N. Beisert, R. Ricci, A. A. Tseytlin, and M. Wolf, Dual superconformal symmetry from $\mathrm{AdS}_{5} \times S^{5}$ superstring integrability, Phys. Rev. D 78, 126004 (2008).

[43] N. Beisert, T-duality, dual conformal symmetry and integrability for strings on $\mathrm{AdS}_{5} \times S^{5}$, Fortschr. Phys. 57, 329 (2009).

[44] L. J. Dixon, J. M. Drummond, and J. M. Henn, Bootstrapping the three-loop hexagon, J. High Energy Phys. 11 (2011) 023.

[45] L. J. Dixon, J. M. Drummond, M. von Hippel, and J. Pennington, Hexagon functions and the three-loop remainder function, J. High Energy Phys. 12 (2013) 049. 
[46] L. J. Dixon and M. von Hippel, Bootstrapping an NMHV amplitude through three loops, J. High Energy Phys. 10 (2014) 065.

[47] S. Caron-Huot, L. J. Dixon, A. McLeod, and M. von Hippel, Bootstrapping a Five-Loop Amplitude Using Steinmann Relations, Phys. Rev. Lett. 117, 241601 (2016).
[48] L. Dixon, A. McLeod, and M. Wilhelm, A three-point form factor through five loops, arXiv:2012.12286.

[49] O. Aharony, O. Bergman, D. L. Jafferis, and J. Maldacena, $\mathcal{N}=6$ superconformal Chern-Simons-matter theories, M2branes and their gravity duals, J. High Energy Phys. 10 (2008) 091. 